\title{
Emerging Methods of Auction Design in Social Networks
}

\author{
Yuhang Guo and Dong Hao* \\ University of Electronic Science and Technology of China \\ guoyuhang@std.uestc.edu.cn, haodong@uestc.edu.cn.
}

\begin{abstract}
In recent years, a new branch of auction models called diffusion auction has extended the traditional auction into social network scenarios. The diffusion auction models the auction as a networked market whose nodes are potential customers and whose edges are the relations between these customers. The diffusion auction mechanism can incentivize buyers to not only submit a truthful bid, but also further invite their surrounding neighbors to participate into the auction. It can convene more participants than traditional auction mechanisms, which leads to better optimizations of different key aspects, such as social welfare, seller's revenue, amount of redistributed money and so on. The diffusion auctions have recently attracted a discrete interest in the algorithmic game theory and market design communities. This survey summarizes the current progress of diffusion auctions.
\end{abstract}

\section{Introduction}

Auction design is a common paradigm and a successful application of market design. Now it has become a representative interface integrating economics and artificial intelligence. Convening a larger group of customers in an auction may possibly increase not only the seller's revenue but also the social welfare. However, classic auction models focus on implementing desired social choices in a fixed group of bidders who can be directly informed by the seller. They do not take the underlying social network among the agents into account. On the contrary, in any real markets, the social and economic relations between entities play an important role.

The role of social networks in auctions is twofold. First, an agent's preference for the selling item is inherently associated with her position in the social network. More importantly, it is the social networks where the sale information spreads and where the commodities flow. That is, the crowds and markets are networked. Without careful consideration of social networks, the sale may be blocked within a local community, leaving many high valuation buyers excluded from the sale.

\footnotetext{
${ }^{*}$ corresponding author.
}

As a result, the auctioneer's revenue and the social welfare can only be locally optimized.

The main difficulty for the auction mechanism to convene more buyers lies in the conflict that although the seller wishes to attract more people to join the auction in order to increase her revenue, the buyers have no incentive to bring more competitors into the auction. This essentially reflects the conflict between the system's optimality and the individuals' selfinterests. To tackle this general problem, in recent years, the diffusion auction has been proposed. Diffusion auction consists of two components: agents' spontaneous expansion of the market and the seller's implementation of allocation and pricing in the expanding market. Since diffusion auction design can convene more participants than traditional mechanism design, it can simultaneously improve the social welfare and the seller's profit, which is known to be a difficult objective. Furthermore, with the development of online social network technology, the interaction between agents is becoming easier, faster and broader, making diffusion auction design more realistic. This survey gives a comprehensive survey of current progress of diffusion auction design.

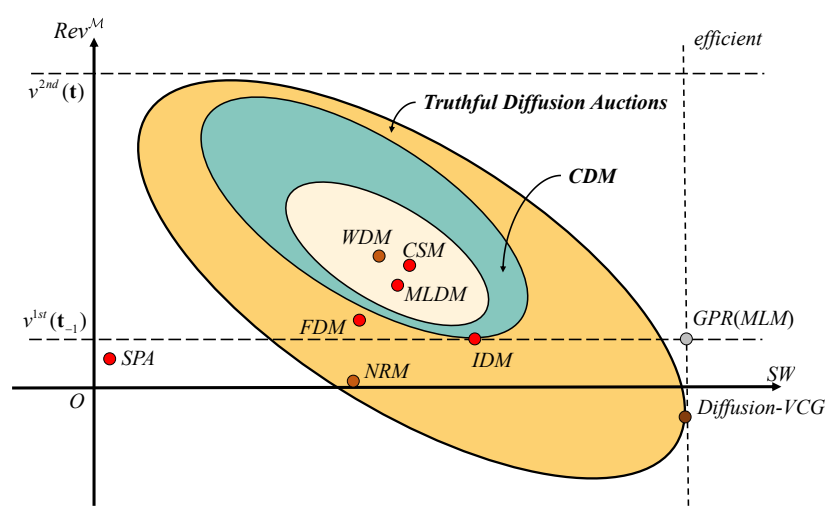

Figure 1: Research map of representative diffusion auction mechanisms under a certain social network. The $x$-axis is the value of social welfare and the $y$-axis is seller's revenue. Each circle is a certain class of diffusion auction mechanisms. Each dot is a specific auction mechanism.

For auction design, efficiency and the seller's revenue are two central criteria, but usually they conflict with each other. With the new concepts and models, various novel diffusion 
auction mechanisms have aligned the optimization of these two criteria in different ways. We illustrate the research map of the most representative mechanisms of diffusion auction in Figure 1. The traditional second-price auction (SPA) sells the item in a local market with relatively low efficiency and revenue. The pioneer works of the diffusion auction [Li et al., 2017; Lee, 2017] have proved that classic VCG mechanism [Vickrey, 1961; Clarke, 1971; Groves, 1973] can be naturally extended to social network scenarios but is not budget balanced. Especially, Li et al. [2017] showed that VCG could incur the seller with a large deficit in extreme network cases. Lee [2017] analysed the conditions for VCG's deficit. Based on these observations of VCG's shortcoming in social networks, Li et al. [2017] further proposed a formal method IDM for modeling the information diffusion and designed the very first incentive-compatible diffusion auction. Lee [2017] proposed another diffusion mechanism MLM which is efficient, has equal revenue with IDM but can not elicit truthfulness. The yellow region in Figure 1 circles the domain of all strategyproof diffusion auctions, with the diffusion version of VCG on a border where the efficiency is maximized but the revenue could even be negative. After these two initial works, CSM [Li et al., 2018] extended IDM into economic networks. Then, CDM and WDM [Li et al., 2019] formulated solutions for unweighted and weighted networks respectively. Also, FDM [Zhang et al., 2020b] and NRM [Zhang et al., 2020c] studied redistribution issues via social networks. Besides these diffusion auction models, Influence Maximization (IM) is a large branch of works investigating how to find influential nodes and how to maximize information propagation [Kempe et al., 2003; Chen et al., 2009; Banerjee et al., 2019]. However, for auction in social networks, an agent needs to reason about her strategy which is a couple of bid and diffusion actions. The IM methods are not applicable for this complex market.

Section 2 explains the fundamental concepts and model of diffusion auctions. Section 3 clarifies and compares diffusion auction mechanisms under different scenarios. Section 4 further introduces incentive design for spontaneous information diffusion under various scenarios other than auction. Section 5 concludes this survey and shows future directions.

\section{Modeling of Diffusion Auction}

\subsection{Networked Auction and Information Diffusion}

A social network can be regarded as a directed graph $G=$ $(N, E)$, in which the nodes $N$ represent agents and the edges $E$ represent the social links between agents. Let $N=$ $\{1, \ldots, n\}$ denote the agent set. For each $i \in N$, there is a set of neighbors $r_{i} \subseteq N \backslash\{i\}$. Agent $i$ can directly communicate with her neighbors, but cannot communicate with other agents. Assume in this social network, there is a seller $s$ who has items to sell and all other agents $i \in N \backslash\{s\}$ are potential buyers of this item. At the very beginning, only the seller's neighbors know the sale and the auction is run only among this local community. This is the general assumption of classic auction theory. On the contrary, the diffusion auction considers a more realistic scenario where after knowing the sale, agents may possibly share this sale information with their neighbors.

Assume $v_{i}$ is $i$ 's valuation of the item and $r_{i} \in \mathcal{P}\left(r_{i}\right)$ is her neighbor set where $\mathcal{P}\left(r_{i}\right)$ is the power set. Agent's type or action is defined by a tuple $t_{i}=\left(v_{i}, r_{i}\right)$ and the type profile is $\mathbf{t}=\left(t_{1}, \cdots, t_{n}\right)$. The type profile except agent $i$ is $\mathbf{t}_{-i}=\left\{t_{1}, \cdots, t_{i-1}, t_{i+1}, \cdots, t_{n}\right\}$. Thus the type of each agent has been expanded from classic single dimensional valuation to a non-typical multi-dimensional structure where one dimension is the valuation and the other dimensions are neighbors. The type space is $T_{i}: V_{i} \times \mathcal{P}\left(r_{i}\right)$ and the type profile space is $T=\times T_{i}$. For any two different types $t_{i}^{1}=\left(v_{i}^{1}, r_{i}^{1}\right)$ and $t_{i}^{2}=\left(v_{i}^{2}, r_{i}^{2}\right)$, the diffusion auctions assume $t_{i}^{1} \succ t_{i}^{2}$ holds iff $v_{i}^{1} \geq v_{i}^{2} \wedge r_{i}^{1} \subseteq r_{i}^{2}$, i.e. reporting a higher bid and diffusing information to fewer neighbors give a bidder a higher probability to win. We say a type profile $\mathbf{t}$ from a subset of agents is feasible when there is a maximum connected subgraph containing the seller and these agents. For example, if all the $n$ agents in the social network know the auction, $\mathbf{t}=\left(t_{1}, \cdots, t_{n}\right)$ is feasible.

A diffusion auction mechanism $\mathcal{M}=(\boldsymbol{\pi}, \mathbf{x})$ consists of an allocation rule $\boldsymbol{\pi}=\left\{\pi_{i}\right\}_{i \in N_{-s}}$ and a payment rule $\mathbf{x}=\left\{x_{i}\right\}_{i \in N_{-s}}$. Similar to conventional auction settings, let $t_{i}^{\prime}=\left(v_{i}^{\prime}, r_{i}^{\prime}\right)$ be bidder $i$ 's reported type where $r_{i}^{\prime} \subseteq r_{i}$ means $i$ diffuses the sale information to a subset of her neighbors $r_{i}^{\prime}$. Given an allocation policy $\boldsymbol{\pi}$ and buyers' reported type profile $\mathbf{t}^{\prime}$, the social welfare of an outcome is defined as $S W\left(\boldsymbol{\pi}, \mathbf{t}^{\prime}\right)=\sum_{i \in N_{-s}} \pi_{i}\left(\mathbf{t}^{\prime}\right) v_{i}$. Given bidder $i$ 's true type $t_{i}=\left(v_{i}, r_{i}\right)$ and reported type profile $\left(t_{i}^{\prime}, \mathbf{t}_{-i}^{\prime}\right)$ and a mechanism $\mathcal{M}=(\boldsymbol{\pi}, \mathbf{x})$, we define bidder $i$ 's quasi-linear utility as $u_{i}\left(t_{i},\left(t_{i}^{\prime}, \mathbf{t}_{-i}^{\prime}\right),(\boldsymbol{\pi}, \mathbf{x})\right)=\pi_{i}\left(t_{i}^{\prime}, \mathbf{t}_{-i}^{\prime}\right) v_{i}-x_{i}\left(t_{i}^{\prime}, \mathbf{t}_{-i}^{\prime}\right)$. From the prospective of seller $s$, her utility is defined as $\operatorname{Rev}^{\mathcal{M}}\left(\mathbf{t}^{\prime}\right)=\sum_{i \in N_{-s}} x_{i}\left(t_{i}^{\prime}, \mathbf{t}_{-i}^{\prime}\right)$.

Next, some concepts used to assess the properties of diffusion mechanisms will be given. First of all, efficiency: Given a diffusion mechanism $\mathcal{M}=(\boldsymbol{\pi}, \mathbf{x})$, we called one allocation policy $\boldsymbol{\pi}^{*}$ is efficient iff for any $\mathbf{t}^{\prime}, \boldsymbol{\pi}^{*} \in$ $\arg \max _{\boldsymbol{\pi}^{\prime}} S W\left(\boldsymbol{\pi}^{\prime}, \mathbf{t}^{\prime}\right)$. Taking single unit auction as an example, efficient allocation policy implies assigning the commodity to the buyer with the highest bid. Secondly, weakly budget balanced: one diffusion mechanism $\mathcal{M}=(\boldsymbol{\pi}, \mathbf{x})$ is weakly budget balanced if for any $\mathbf{t}^{\prime}, \operatorname{Rev} v^{\mathcal{M}}\left(\mathbf{t}^{\prime}\right)=$ $\sum_{i \in N_{-s}} x_{i}\left(t_{i}^{\prime}, \mathbf{t}_{-i}^{\prime}\right) \geq 0$ always holds. That is to say the seller $s$ will never incur a deficit.

\subsection{Incentive Compatibility in Diffusion Auction}

One key feature in auctions is incentive compatibility, an auction mechanism is called incentive-compatible (IC) if every bidder can achieve her best outcome just by acting according to their true type. Another significant concept in auctions is individual rational (IR), which means any bidder will never suffer from a loss as long as she truthfully reports her valuation. The well-known Myerson's Lemma [Myerson, 1981] gave a framework for finding IR and IC mechanisms in a single-parameter environment as follows.

Theorem 1. (Myerson's Lemma). Fix a single-parameter auction environment: An allocation rule $\pi$ is implementable iff it is value-monotonic, and if the allocation rule $\pi$ is valuemonotonic, then there is a unique payment rule $\mathrm{x}$ for which 


\begin{tabular}{llll}
\hline $\mathcal{M}$ & Allocation: $\boldsymbol{\pi}$ & Payment: $\mathbf{x}$ & $R e v^{\mathcal{M}}$ \\
\hline VCG & $S W^{*}\left(\mathbf{t}^{\prime}\right)$ & $S W\left(\mathbf{t}_{-i}^{\prime}\right)-\left(S W\left(\mathbf{t}^{\prime}\right)-\pi_{i} v_{i}\right)$ & $\sum_{i \in C_{w} \backslash\{1\}}\left(v_{-i}^{*}-v_{N_{-s}}^{*}\right)+v_{-1}^{*}$ \\
IDM & $S W^{*}\left(\mathbf{t}_{-(i+1)}^{\prime}\right)$ & $S W\left(\mathbf{t}_{-i}^{\prime}\right)-\left(S W\left(\mathbf{t}_{-(i+1)}^{\prime}\right)-\pi_{i} v_{i}\right)$ & $v_{-1}^{*}$ \\
CDM & $S W^{*}\left(\mathbf{t}_{-\alpha_{i}}^{\prime}\right)$ & $S W\left(\mathbf{t}_{-i}^{\prime}\right)-\left(S W\left(\mathbf{t}_{-\alpha_{i}}^{\prime}\right)-\pi_{i} v_{i}\right)$ & $\sum_{i \in C_{w} \backslash\{1, w\}}\left(v_{-(i+1)}^{*}-v_{-\alpha_{i}}^{*}\right)+v_{-1}^{*}$ \\
FDM & $S W^{*}\left(\mathbf{t}_{-(i+1) \cup M_{i, i+1}}^{\prime}\right)$ & $S W\left(\mathbf{t}_{-i}^{\prime}\right)-\left(S W\left(\mathbf{t}_{-(i+1) \cup M_{i, i+1}}^{\prime}\right)-\pi_{i} v_{i}\right)-R_{i}$ & $R_{s}+v_{-1}^{*}$ \\
NRM & $S W^{*}\left(\mathbf{t}_{-T(i)}^{\prime}\right)$ & $S W\left(\mathbf{t}_{-i}^{\prime}\right)-\left(S W\left(\mathbf{t}_{-T(i)}^{\prime}\right)-\pi_{i} v_{i}\right)-R_{i}^{\prime}$ & $0\left(\left|N_{-s}\right| \rightarrow+\infty\right)$ \\
\hline
\end{tabular}

Table 1: Table for comparing single-item diffusion auctions. Denote $w$ by the winner satisfying $S W^{*}(\cdot)$ function, $C_{w}$ is the critical diffusion sequence and only those bidders in $C_{w}$ have non-zero payment. $\alpha_{i}$ is an edge set satisfying three properties in CDM. $M_{i, i+1}$ in FDM represents the set of nodes connecting critical node $i$ and $i+1 . R_{i}$ and $R_{i}^{\prime}$ are different reward functions in FDM and NRM.

$\mathcal{M}=(\boldsymbol{\pi}, \mathbf{x})$ is IR and IC, the winner takes the item and pays the critical bid while all other losers' payment is zero.

When taking social networks and information diffusion into account, Myerson's Lemma no longer applies because strategic diffusion affects agents' critical bids and payment. Also, the definitions of IR and IC change in diffusion auctions: IR represents no matter how one bidder diffuses the sale information, reporting true valuation ensures her nonnegative returns while IC is modified as truthfully reporting her valuation and propagating sale information to all her neighbors will always maximize her utility.

To formulate IC for diffusion auctions, Li et al. [2020b] decouples the payment $x_{i}$ as $x_{i}\left(\mathbf{t}^{\prime}\right)=\pi_{i}\left(\mathbf{t}^{\prime}\right) \tilde{x}_{i}\left(\mathbf{t}^{\prime}\right)+(1-$ $\left.\pi_{i}\left(\mathbf{t}^{\prime}\right)\right) \bar{x}_{i}\left(\mathbf{t}^{\prime}\right)$. Here $\tilde{x}_{i}$ represents bidder $i$ 's payment for winning the item and $\bar{x}_{i}$ represents her payment for losing the item which can be intuitively considered as the reward for diffusion. They show that to ensure a diffusion auction to be IC, the allocation rule should be monotone over the bid; the decoupled payments should be independent of the bid; for a fixed $r_{i}$, the winning payment and losing payment should be monotone over the diffusion effort and the difference between them should equal the bidder's critical bid. More formally, they proved the following theorem.

Theorem 2. A single item diffusion auction is dominantstrategy incentive-compatible iff $(A) \boldsymbol{\pi}$ is value-monotonic; (B) $\tilde{x}_{i}$ and $\bar{x}_{i}$ are bid-independent; (C) $\tilde{x}_{i}\left(r_{i}\right)-\bar{x}_{i}\left(r_{i}\right)=$ $v_{i}^{*}\left(r_{i}\right) ;(D) \tilde{x}_{i}$ and $\bar{x}_{i}$ are diffusion-monotonic; $(E) \bar{x}_{i}(\emptyset) \leq 0$.

After identifying the truthful diffusion auctions, they also show that the optimal revenue for seller $s$ under the truthful diffusion auctions can be calculated as:

$$
\operatorname{Rev}(\mathbf{t})=\sum_{i \in N_{-s}}\left[v_{i}^{*}(\emptyset)-v_{i}^{*}\left(r_{i}\right)\left(1-\pi_{i}(\mathbf{t})\right)\right] .
$$

From the perspective of the seller $s$, if bidder $i$ is the winner, her optimal payment is the critical bid when she does not diffuse: $v_{i}^{*}(\emptyset)$, otherwise, the optimal payment should be the difference between critical bid under no diffusion and truthful diffusion: $v_{i}^{*}(\emptyset)-v_{i}^{*}\left(r_{i}\right)$.

\section{Diffusion Auction Mechanisms}

\subsection{Diffusion Auction in Unweighted Network}

The initial work of diffusion auction in [Li et al., 2017] assumes the diffusion cost is negligible, which can be captured by unweighted networks. They proposed the information diffusion mechanism (IDM), which firstly introduced the concepts of critical diffusion nodes and critical diffusion sequences. Then the diffusion process in the social network can be captured by a tree on which the auction is conducted. These concepts and methods are also utilized in the follow-up works of diffusion mechanisms. Consider two bidders $i$ and $j, i$ is $j$ 's critical diffusion node means $j$ could not participate in this auction without $i$ 's diffusion and all of $j$ 's critical diffusion nodes form her critical diffusion sequence.

Li et al. [2019] further investigated a class of diffusion auction mechanisms under social networks, which is named as critical diffusion mechanisms (CDM). Also, they found that IDM is a special case of this newly discovered set of auction mechanisms, which has the lowest seller's revenue in this set. CDMs rely on the critical diffusion nodes and sequence. However, according to the theorem of small-world [Amaral et al., 2000], cut-points rarely exist in large well-connected real social network. Thus under CDM, the majority of normal nodes may not have strong incentives to diffuse.

Inspired by the redistribution mechanisms in [Cavallo, 2006; Guo and Conitzer, 2009; Guo, 2012], Zhang et al. [2020c] proposed the fair diffusion mechanism (FDM) which benefit not only cut nodes but also those nodes who have made contributions for the connection from the seller to the winner. Later, Zhang et al. [2020b] investigated a redistribution mechanism on networks called network-based redistribution mechanism (NRM) for more efficient resource allocation. NRM has been proved to be IR, IC and asymptotically budget-balanced. Another work [Zhang et al., 2019] in single-unit diffusion auctions without diffusion allies the information diffusion model into fixed-price mechanism design and studied fixed price diffusion mechanism (FPDM), which promises the seller $1 / 2$ optimal revenue.

Table 1 summarizes the allocation rules, payment rules and revenues of all single-item diffusion auction mechanisms. We make comparisons of the above diffusion auction mechanisms in the example network in Figure 2 and present the results among these mechanisms in Table 2. With the social network in Figure 2, initially, the seller $s$ could only sell one commodity among agents $\{a, b, c\}$. Bidder $b$ would win and pay 2 under a second-price auction. However, when truthful diffusion mechanisms are applied, all participants are willing to propagate the sale information. Thus more potential bidders such as $\{k, m, o\}$ will join.

Because CDM represents a family of diffusion mechanisms, here we take $\alpha_{i}$ as the minimum cutting edges subset 
which makes the next critical diffusion node can not attend the auction as one special case. From Table 2, we can see different single unit auction mechanisms have different advantages. VCG guarantees allocation efficiency but not promises the seller's revenue; IDM is a particular case of CDM. The family of these mechanisms sacrifice efficiency but benefit the seller with higher profit. FDM and NRM aim for rewarding not only cut nodes but also those making contributions for information propagation, thus benefiting more reasonable resource allocation in society.

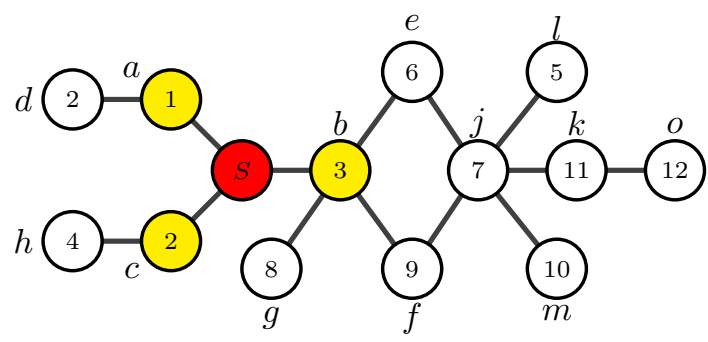

Figure 2: Instance of auction in social networks. The colored nodes constitute a local community where the classic auction is conducted. Without information diffusion, the agents with high valuations can not join the sale.

\begin{tabular}{lllll}
\hline $\mathcal{M}$ & Winner & Rewarded bidders & $S W$ & $\operatorname{Rev}^{\mathcal{M}}$ \\
\hline SPA & $b(2)$ & $\emptyset$ & 3 & 2 \\
VCG & $o(11)$ & $b(-8), j(-3), k(-2)$ & 12 & -2 \\
IDM & $k(10)$ & $b(-5), j(-1)$ & 11 & 4 \\
CDM & $k(10)$ & $b(-4), j(-1)$ & 11 & 5 \\
FDM & $k(10)$ & $b(-4), e\left(-\frac{1}{3}\right), j(-1)$ & 11 & $\frac{14}{3}$ \\
NRM & $k(10)$ & $a\left(-\frac{8}{13}\right), b\left(-\frac{18}{13}\right), c\left(-\frac{4}{13}\right)$ & 11 & $\frac{61}{26}$ \\
& & $e\left(-\frac{5}{8}\right), f\left(-\frac{1}{2}\right), g\left(-\frac{5}{8}\right)$ & & \\
& & $j\left(-\frac{5}{2}\right), l\left(-\frac{1}{4}\right)$ & \\
\hline
\end{tabular}

Table 2: Numerical results for different diffusion auctions run in the network given in Figure 2. Numbers in the parentheses after the nodes are payments. Positive value means the agent pays to the seller while negative value means the seller pays to the agent.

\subsection{Diffusion Auction in Weighted Network}

In many cases, we assume the information dissemination is cost-free. However, it is often the scenario that information diffusion in social networks requires fees. Leduc et al. [2017] focused on referral payments in social networks. Condorrelli et al. [2018] studied such a setting where the seller sells items to buyers only through intermediaries who can be either merchants or referrals.

An example of weighted economic networks is shown in Figure 3. In such economic networks, all the potential buyers consist of the buyers set $B$; denote $I$ by the intermediaries set and $N=B \cup I \cup\{s\}$ by all agents set. Buyer $i$ 's type is defined as $t_{i}=\left(v_{i}, \emptyset\right)$, where $v_{i}$ is her valuation for the item and $r_{i}=\emptyset$ means that she is not able to diffuse the sale information; intermediary $k$ 's type is defined as $t_{k}=\left(c_{k}, r_{k}\right)$, where $c_{k}$ is the cost she charges for one successful trading and $r_{k}$ is the set of her customers.

Li et al. [2018] is the first to explore auctions in such weighted economic networks. Their customer sharing mechanism (CSM) allocates the item to a potential buyer whose trading path would maximize the social welfare. One trading path consists of a seller $s$, a final buyer $i \in B$ and a series of intermediaries $k \in I$. Social welfare in economic networks is different from that in social networks without transfer cost and it is redefined as: $S W=\pi_{i} v_{i}-\sum_{k \in I} c_{k}$. CSM charges the buyer $i$ VCG's payment: the social welfare decrease of the other agents caused by $i$ 's participation, and charges the intermediary $k$ the social welfare decrease correlated with their threshold neighbourhood set $r_{k}^{*}$. $r_{k}^{*}$ represents a minimum subset of $r_{k}$ where $k$ 's diffusion strategy changing from $r_{k}$ to $r_{k} \backslash r_{k}^{*}$ could change the winner under efficient allocation.

Li et al. [2020a] extended market resource allocation under particular economic networks into a more general setting where intermediaries can not only diffuse sale information to those bidders but also can be potential buyers. Still, all bidders' access to the sale is from the seller and intermediaries' invitation. Their mechanisms Single-Level Diffusion Mechanism (SLDM) and Multi-Level Diffusion Mechanism (MLDM) applies to the single level distribution market scenarios where all intermediaries are connected to the seller and multiple-level markets where intermediaries diffuse the information in a tree structure respectively.

In the above economic networks, information flows in one direction from the seller through intermediaries to buyers, which means those buyers cannot diffuse sale information. They can never participate in these auctions without the seller and the intermediaries. Li et al. [2019] generalized economic networks to a weighted graph where all participants could bid and propagate sale information and weights of edges represent transfer cost between agents. Further, they illustrated the differences between auctions via unweighted networks and weighted ones, explained why all previous mechanisms cannot be applied to a weighted graph scenario and proposed weighted diffusion mechanism (WDM), which can deal with this challenging problem. They illustrated that the minimum cost allocation path conflicts with the critical propagation path in weighted networks. WDM allocates along the minimum cost trading path and assigns the item to the first bidder $i$ whose bid is highest when her diffusion strategy changes $r_{i}$ to $r_{i} \backslash \gamma_{i}^{*}$. Here $\gamma_{i}^{*}$ is a special edge set containing $i$ and the concatenated edges of those non-terminal nodes in $r_{i}$. All agents

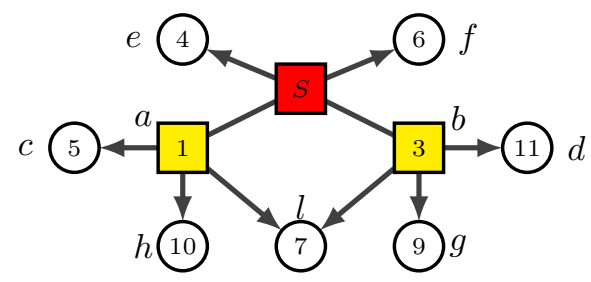

Figure 3: Economic network example: The red rectangle node $s$ is the seller, yellow rectangle nodes $\{a, b\}$ are the intermediaries, and all other circle nodes $\{c, d, e, f, g, h, l\}$ are those potential buyers. Numbers in rectangle nodes represent information diffusion cost. 
in minimum cost trading path pay $S W^{*}\left(t_{-i}^{\prime}\right)-S W^{*}\left(t_{-\gamma_{i}^{*}}^{\prime}\right)$ and the winner undertakes the critical bid that could beat all nodes before her in the trading path with minimum cost.

Comparing diffusion auctions in weighted networks and unweighted networks, without diffusion cost, we focus on the critical diffusion sequences and allocate the item to the winner in any trading path. However, when considering cost, it changes a lot since the cost is related to efficiency, bidders' allocation and seller's revenue, thus all these with-cost diffusion mechanisms focus more on the trading path with minimum cost rather than the critical diffusion sequence in costfree diffusion auctions. This is the most significant difference between these two scenarios.

\subsection{Non-Truthful Diffusion Auction}

From the well-known work [Green and Laffont, 1979], we can know that efficiency and weakly budget-balance for designing mechanisms in general quasi-linear domains cannot be achieved at the same time. This is also true in diffusion auctions. Some diffusion mechanisms sacrifice efficiency to ensure weakly budget-balance while some others maintain efficiency with a deficit, such as VCG with diffusion. However, there exist another branch of diffusion auctions where IC is sacrificed, ensuring efficiency and weakly budget-balance.

The very first work in this domain is from MLM[Lee, 2017]. Based on the fact that VCG diffusion mechanism is efficient, IC, IR but not budget feasible, they proposed their multilevel mechanisms (MLM). In a single-item diffusion auction, MLM allocates the item to the highest bidder. Its payment rule makes some improvement on the VCG payment, from individual externality to groupwise externality. The specific differences between individual externality $\left(x_{i}^{\mathrm{ie}}\right)$ and groupwise externality $\left(x_{i}^{\mathrm{ge}}\right)$ are as follows:

$$
\begin{aligned}
x_{i}^{\mathrm{ie}} & =S W^{*}\left(\mathbf{t}_{-i}^{\prime}\right)-\left(S W^{*}\left(\mathbf{t}^{\prime}\right)-\pi_{i} v_{i}\right), \\
x_{i}^{\mathrm{ge}} & =S W^{*}\left(\mathbf{t}_{-i}^{\prime}\right)-\left(S W^{*}\left(\mathbf{t}^{\prime}\right)-\sum_{j \in T(i)} \pi_{j} v_{j}\right) .
\end{aligned}
$$

Note $T(i)$ represents the set of bidders under $i$ 's critical diffusion sub-tree. Further, the payment rules of VCG equals $x_{i}^{\text {ie }}$ and MLM can be illustrated as follows:

$$
x_{i}^{\mathrm{MLM}}=x_{i}^{\mathrm{ge}}-\sum_{k \in C(i)} x_{k}^{\mathrm{ge}} .
$$

Note that $C(i)$ represents the set of $i$ 's immediate children, specifically, all agents who are $i$ 's first-order neighbors while $i$ is their critical diffusion node.

MLM is efficient, weakly budget balanced, IR but not IC. Buyers could manipulate the auction by misreporting higher bids. Some analysis and improvements on the MLM have been made in [Jeong, 2020] and [Jeong and Lee, 2020]. Jeong [2020] introduced referral monotonicity: when all other bidders' types are fixed, the utility will never decrease with her diffusion strategy for every potential buyer, then they proposed the Referrer's Dilemma: although more diffusion brings non-decrease profit from individuals, when all bidders try to propagate information, they are weakly worse off but the seller weakly increases her revenue from a global perspective. They examined which mechanisms are subject to this dilemma and the impact of false-name attacks and collisions on these mechanisms. Jeong and Lee [2020] renamed the multilevel mechanism as groupwise-pivotal referral mechanism (GPR) and proved it to be groupwise collusion-proof (any complicity in groups can not improve utility) and Sybilproof (preventing false-name manipulation).

\subsection{Multi-Unit Diffusion Auction}

A classic multi-unit auction allocates multiple homogeneous items to bidders. Multi-unit auctions are widely used in different social sectors for electromagnetic spectrum[Cramton, 1997; Cramton and Schwartz, 2000], electricity distribution, government securities, etc. When extending multi-unit auctions into social networks, it becomes a very complicated decision-making problem since ancestor bidders can control items passed to their children, influencing their payments. At the same time, they can change their peers' payments without changing their allocation and payments.

Even in multi-unit auctions with single-unit demand where many results [Krishna, 2009] in single-item auctions can be extended, it becomes quite complex in the context of diffusion. Zhao et al. [2018] firstly proposed the general information diffusion mechanism (GIDM) to solve multi-unit diffusion auctions with single-unit demand. They used a critical diffusion tree based on the critical diffusion sequence in IDM and divided it into some sub-markets. Considering one $k$ unit diffusion auction with single-unit demand, all the top- $k$ bidders' critical diffusion sequences consist of the critical diffusion tree and whether top- $k$ bidders can get one item or not depends on their ancestors. If one ancestor is eligible for an item, then the original top- $k$ node with the lowest bid under the ancestor's sub-tree will cede the item. GIDM is the first attempt to tackle the multi-unit diffusion auction design and it inspires a few follow-up works on this complicated task.

Takanashi et al. [2019] studied Generalized Aligned Path Graph Mechanism (GAPG) for multi-unit auctions with decreasing marginal utility, which is IC, IR but not weakly budget balanced. Further, Distance-based Network Auction Mechanism (DNA-MU) for multi-unit diffusion auction with single-item demand was proposed in [Kawasaki et al., 2020]. It allocates items in the order of distance from the seller to buyers and one buyer $i$ is qualified to win an item when her reported price satisfies $v_{i}^{\prime} \geq v^{k}\left(\mathbf{t}_{-\{i\} \cup W}^{\prime}\right)$. Here $W$ is the winner set and once a buyer is assigned one item, she will be added into $W$. The payment of winner $i$ is this critical bid $v^{k}\left(\mathbf{t}_{-\{i\} \cup W}^{\prime}\right)$. Whenever an item is assigned out, the total number of items $k$ is self-reduced by 1 . The whole process finishes when all $k$ items are allocated. DNA-MU mechanism is proved to be IR and weakly IC, which means agents have no direct incentives to invite their friends to join since they can not directly gain reward from diffusion. More detailed analysis of efficiency and revenue are finely illustrated in this work. It gave a good viewpoint that the quantified network structure criteria such as path length, degree, clustering coefficient can be utilized for the diffusion auction design.

Condorrelli et al. [2017] studied bilateral trading in networks. Xu et al. [2019] introduced networked double auction with multiple sellers and buyers existing in their problem 
setting. Their mechanism Double Network Auction Mechanism (DNAM) combines McAfee's mechanism [McAfee, 1993] with VCG mechanism. DNAM can be degenerated into single-unit cases since their sub-markets are independent.

Researches on multi-unit diffusion auctions are incomprehensive yet and there still remains many challenging problems. For instance, monotonic allocation rules involving diffusion is difficult to identify, how to restrict the bidders' action space to simplify the outcome space is still unclear, etc.

\section{Diffusion Incentive Design beyond Auctions}

There are several application directions where incentive matters but there is no bid for purchasing. Such applications include crowdsourcing, matching, voting and so on.

Crowdsourcing [Howe, 2006] is a collaboration model where some institutions try to attract potential employees to solve a colossal task, which takes the form of peerproduction. All the agents involved solve the job collaboratively (e.g. LEGO Ideas, Amazon Mechanical Turk). In traditional crowdsourcing problems, the requester pays not only employees but also third-party platforms with a high cost; the quality of sourcing from the paid platforms may not be guaranteed. However, employees' recruitment can be done spontaneously when considering the collaboration via social networks. Since employees are rewarded directly from the requester, the quality and flexibility are also improved.

DARPA 2009 red balloon challenge is a typical example in this field. This challenge awards the first team who submits positions of all ten weather red balloons in the continental United States. Pickard et al. [2011] gave the winner solution for this task: recursive incentive mechanism (RIM), which diffuses task information through social networks and provides incentives for individuals who give solutions and make recruitment. The reward takes a bottom-up cascading form. Cebrain et al. [2012] provided a split contract mechanism in the same setting. Each individual receives rewards from her parents and decides the reward offer to her children nodes in their mechanism. Emek et al. [2011] deals with multi-level marketing mechanisms in which small rewards are allocated for preventing false-name manipulations. Zhang et al. [2020d] studied a question answering problem. They characterized the incompatibility between Sybilproofness and collusion-proofness under strong IR assumption and proposed Double Geometric Mechanism (DGM), which is Sybil-proof and 2-collusion-proof.

Zhang et al. [2020a] focused on another crowdsourcing instance called data acquisition, where a requester acquires useful data (e.g. images, corpus) via social networks. Their target is to incentivize all agents to provide all their valid data and inviting their neighbors. their novel crowdsourcing diffusion mechanism rewards one agent for her contributions to data and diffusion. Shapley value describes how to distribute both gains and costs to several agents working in a coalition[Roth, 1988]. They modified it into the layered Shapley value since the traditional form discourages agents from inviting their friends. Agents with the same depth in the diffusion network are treated as one group and their reward equals the marginal contribution for their participation. Also, information entropy[Shannon, 1948] is used as a valuation function in layered Shapley value.

Zhang et al. [2020] extended information diffusion settings into a cooperative game. To incentivize agents' diffusion, they combined weighted Shapley value[Radzik, 2012] which is applied in cooperative asymmetry game, with permission structure[Gilles et al., 1992] which requires that some agents could join in the coalition only if getting permission from other agents. Shi et al. [2020] focused on an information diffusion game via social networks where one requester wants to spread information via social networks, paying the participants for their diffusion with a fixed budget. Their scheme contains two stages: the first is hierarchizing the entire diffusion tree and deciding the sum of rewards for each layer based on the total budget while the second is determining rewards for agents in each layer respectively.

Social choice problems like matching, voting, rating under network scenarios are also important research fields. The pioneer work in this thread is from [Grandi et al., 2015], which is later generalized in [Grandi et al., 2017; Grandi et al., 2020]. They studied the opinion diffusion problem with multiple agents in social network settings and showed how one agent is affected by the opinions of those agents that she trust, like relatives, neighbors or good friends. The social network structure is a key component in analyzing and modeling the trust and reputation.

Some other works [Zheng et al., 2020; Kawasaki et al., 2021] focus on the networked house allocation problem. Kawasaki et al. [2021] defined new networked core concepts and illustrated incompatibility between networked core and strategyproofness. They restricted preference into acyclic domain to guarantee stratgyproofness of TTC in networked market and proposed modified TTC under tree networked market.

\section{Discussion}

Past few years have witnessed considerable progress in this emerging networked auction. Generally, we have highlighted single item networked auction design and multi-unit with single demand, diffusion mechanisms focus on incentivizing truthful bidding and inviting neighbors to join, requiring tradeoff between different properties. However, tasks about multi-unit and combinatorial auctions via social networks have not yet been explored clearly. It is also a valuable topic to integrate network structures into property analysis in diffusion mechanisms design. Besides, Sybil-proofness [Todo and Yokoo, 2020] and collusion-proofness in networked auction are both nice directions.

There are other representative scenarios where the incentive design of information diffusion deserves further investigation beyond auction. For example, how to conduct a more credible election or voting [Colley et al., 2020] by encouraging more people to join; how to facilitate a good organ donation [Roth et al., 2004; Roth et al., 2005] and transplantation system in a networked scenario; how to incentivize more people into a rating system; and how to notice more citizens to conduct public opinion surveys. Any other mechanism design scenarios where attracting more participants is needed can also be extended into social network settings. 


\section{References}

[Amaral et al., 2000] Luis A Nunes Amaral, Antonio Scala, Marc Barthelemy, and H Eugene Stanley. Classes of smallworld networks. Proceedings of the national academy of sciences, 97(21):11149-11152, 2000.

[Banerjee et al., 2019] Prithu Banerjee, Wei Chen, and Laks VS Lakshmanan. Maximizing welfare in social networks under a utility driven influence diffusion model. In Proceedings of the 2019 International Conference on Management of Data, pages 1078-1095, 2019.

[Cavallo, 2006] Ruggiero Cavallo. Optimal decision-making with minimal waste: strategyproof redistribution of VCG payments. In Proceedings of the 5th International Conference on Autonomous Agents and MultiAgent Systems, pages 882-889. ACM, 2006.

[Cebrian et al., 2012] Manuel Cebrian, Lorenzo Coviello, Andrea Vattani, and Panagiotis Voulgaris. Finding red balloons with split contracts: robustness to individuals' selfishness. In Proceedings of the forty-fourth annual ACM symposium on Theory of computing, pages 775-788, 2012.

[Chen et al., 2009] Wei Chen, Yajun Wang, and Siyu Yang. Efficient influence maximization in social networks. In Proceedings of the 15th ACM SIGKDD international conference on Knowledge discovery and data mining, pages 199-208, 2009.

[Clarke, 1971] Edward H Clarke. Multipart pricing of public goods. Public choice, pages 17-33, 1971.

[Colley et al., 2020] Rachael Colley, Umberto Grandi, and Arianna Novaro. Smart voting. In Proceedings of the 29th International Joint Conference on Artificial Intelligence, pages 1734-1740, 2020.

[Condorelli et al., 2017] Daniele Condorelli, Andrea Galeotti, and Ludovic Renou. Bilateral trading in networks. The Review of Economic Studies, 84(1):82-105, 2017.

[Condorelli et al., 2018] Daniele Condorelli, Andrea Galeotti, and Vasiliki Skreta. Selling through referrals. Journal of economics \& management strategy, 27(4):669-685, 2018.

[Cramton and Schwartz, 2000] Peter Cramton and Jesse A Schwartz. Collusive bidding: Lessons from the fcc spectrum auctions. Journal of regulatory Economics, 17(3):229-252, 2000.

[Cramton, 1997] Peter Cramton. The fcc spectrum auctions: An early assessment. Journal of Economics \& Management Strategy, 6(3):431-495, 1997.

[Emek et al., 2011] Yuval Emek, Ron Karidi, Moshe Tennenholtz, and Aviv Zohar. Mechanisms for multi-level marketing. In Proceedings of the 12th ACM conference on Electronic commerce, pages 209-218, 2011.

[Gilles et al., 1992] Robert P Gilles, Guillermo Owen, and Rene van den Brink. Games with permission structures: the conjunctive approach. International Journal of Game Theory, 20(3):277-293, 1992.
[Grandi et al., 2015] Umberto Grandi, Emiliano Lorini, and Laurent Perrussel. Propositional opinion diffusion. In Proceedings of the 14th International Conference on Autonomous Agents and Multiagent Systems, page 989-997, 2015.

[Grandi et al., 2017] Umberto Grandi, Emiliano Lorini, Arianna Novaro, and Laurent Perrussel. Strategic disclosure of opinions on a social network. In Proceedings of the 16th Conference on Autonomous Agents and MultiAgent Systems, pages 1196-1204, 2017.

[Grandi et al., 2020] Umberto Grandi, James Stewart, and Paolo Turrini. Personalised rating. Autonomous Agents and Multi-Agent Systems, 34(2):1-38, 2020.

[Green and Laffont, 1979] Jerry R. Green and Jean-Jacques Laffont. Incentives in Public Decision Making. NorthHolland, Amsterdam, 1979.

[Groves, 1973] Theodore Groves. Incentives in teams. Econometrica: Journal of the Econometric Society, pages 617-631, 1973.

[Guo and Conitzer, 2009] Mingyu Guo and Vincent Conitzer. Worst-case optimal redistribution of VCG payments in multi-unit auctions. Games Econ. Behav., 67(1):69-98, 2009.

[Guo, 2012] Mingyu Guo. Worst-case optimal redistribution of VCG payments in heterogeneous-item auctions with unit demand. In Proceedings of the 11th International Conference on Autonomous Agents and Multiagent Systems, pages 745-752, 2012.

[Howe, 2006] Jeff Howe. The rise of crowdsourcing. Wired magazine, 14(6):1-4, 2006.

[Jeong and Lee, 2020] Seungwon Eugene Jeong and Joosung Lee. The groupwise-pivotal referral mechanism: Core-selecting referral strategy-proof mechanism. Social Science Research Network (SSRN) ePrint 3574093, pages 1-39, 2020.

[Jeong, 2020] Seungwon Eugene Jeong. The referrer's dilemma. Social Science Research Network (SSRN) ePrint 3514022, pages 1-25, 2020.

[Kawasaki et al., 2020] Takehiro Kawasaki, Nathanael Barrot, Seiji Takanashi, Taiki Todo, Makoto Yokoo, et al. Strategy-proof and non-wasteful multi-unit auction via social network. In AAAI, pages 2062-2069, 2020.

[Kawasaki et al., 2021] Takehiro Kawasaki, Ryoji Wada, Taiki Todo, and Makoto Yokoo. Mechanism design for housing markets over social networks. In Proceedings of the 20th International Conference on Autonomous Agents and Multiagent Systems, page 692-700. International Foundation for Autonomous Agents and Multiagent Systems, 2021.

[Kempe et al., 2003] David Kempe, Jon Kleinberg, and Éva Tardos. Maximizing the spread of influence through a social network. In Proceedings of the ninth ACM SIGKDD international conference on Knowledge discovery and data mining, pages 137-146, 2003. 
[Krishna, 2009] Vijay Krishna. Auction theory. Academic press, 2009.

[Leduc et al., 2017] Matt V Leduc, Matthew O Jackson, and Ramesh Johari. Pricing and referrals in diffusion on networks. Games and Economic Behavior, 104:568-594, 2017.

[Lee, 2017] Joosung Lee. Mechanisms with Referrals: VCG Mechanisms and Multilevel Mechanisms. Working Papers 2017.27, Fondazione Eni Enrico Mattei, June 2017.

[Li et al., 2017] Bin Li, Dong Hao, Dengji Zhao, and Tao Zhou. Mechanism design in social networks. In $A A A I$, pages 586-592, 2017.

[Li et al., 2018] Bin Li, Dong Hao, Dengji Zhao, and Tao Zhou. Customer sharing in economic networks with costs. In Proceedings of the 27th International Joint Conference on Artificial Intelligence, pages 368-374, 2018.

[Li et al., 2019] Bin Li, Dong Hao, Dengji Zhao, and Makoto Yokoo. Diffusion and auction on graphs. In Proceedings of the 28th International Joint Conference on Artificial Intelligence, pages 435-441, 2019.

[Li et al., 2020a] Bin Li, Dong Hao, Fan Liu, Hui Gao, and Tao Zhou. Information diffusion and revenue optimization in distribution market. IEEE Access, 8:40850-40860, 2020.

[Li et al., 2020b] Bin Li, Dong Hao, and Dengji Zhao. Incentive-compatible diffusion auctions. In Proceedings of the 29th International Joint Conference on Artificial Intelligence, pages 231-237, 2020.

[McAfee, 1993] R Preston McAfee. Mechanism design by competing sellers. Econometrica: Journal of the econometric society, pages 1281-1312, 1993.

[Myerson, 1981] Roger B Myerson. Optimal auction design. Mathematics of operations research, 6(1):58-73, 1981.

[Pickard et al., 2011] Galen Pickard, Wei Pan, Iyad Rahwan, Manuel Cebrian, Riley Crane, Anmol Madan, and Alex Pentland. Time-critical social mobilization. Science, 334(6055):509-512, 2011.

[Radzik, 2012] Tadeusz Radzik. A new look at the role of players' weights in the weighted shapley value. European Journal of Operational Research, 223(2):407-416, 2012.

[Roth et al., 2004] Alvin E Roth, Tayfun Sönmez, and M Utku Ünver. Kidney exchange. The Quarterly journal of economics, 119(2):457-488, 2004.

[Roth et al., 2005] Alvin E Roth, Tayfun Sönmez, and M Utku Ünver. Pairwise kidney exchange. Journal of Economic theory, 125(2):151-188, 2005.

[Roth, 1988] Alvin E Roth. The Shapley value: essays in honor of Lloyd S. Shapley. Cambridge University Press, 1988.

[Shannon, 1948] Claude E Shannon. A mathematical theory of communication. The Bell system technical journal, 27(3):379-423, 1948.
[Shi et al., 2020] Haomin Shi, Yao Zhang, Zilin Si, Letong Wang, and Dengji Zhao. Maximal information propagation with budgets. In 24th European Conference on Artificial Intelligence, ECAI 2020, including 10th Conference on Prestigious Applications of Artificial Intelligence, PAIS 2020, pages 211-218, 2020.

[Takanashi et al., 2019] Seiji Takanashi, Takehiro Kawasaki, Taiki Todo, and Makoto Yokoo. Efficiency in truthful auctions via a social network. arXiv preprint arXiv:1904.12422, 2019.

[Todo and Yokoo, 2020] Taiki Todo and Makoto Yokoo. Split manipulations in cost sharing of minimum cost spanning tree. In 24th European Conference on Artificial Intelligence, ECAI 2020, including 10th Conference on Prestigious Applications of Artificial Intelligence, PAIS 2020, pages 219-226, 2020.

[Vickrey, 1961] William Vickrey. Counterspeculation, auctions, and competitive sealed tenders. The Journal of $f$ nance, 16(1):8-37, 1961.

[Xu et al., 2019] Junping Xu, Xin He, and Dengji Zhao. Double auction design on networks. In Proceedings of the First International Conference on Distributed Artificial Intelligence, pages 1-6, 2019.

[Zhang and Zhao, 2020] Yao Zhang and Dengji Zhao. Incentives to form larger coalitions when players have the power to choose. arXiv preprint arXiv:2011.09049, 2020.

[Zhang et al., 2019] Tianyi Zhang, Dengji Zhao, Wen Zhang, and Xuming He. Fixed-price diffusion mechanism design. arXiv preprint arXiv:1905.05450, 2019.

[Zhang et al., 2020a] Wen Zhang, Yao Zhang, and Dengji Zhao. Collaborative data acquisition. In Proceedings of the 19th International Conference on Autonomous Agents and Multiagent Systems, pages 1629-1637, 2020.

[Zhang et al., 2020b] Wen Zhang, Dengji Zhao, and Hanyu Chen. Redistribution mechanism on networks. In Proceedings of the 19th International Conference on Autonomous Agents and MultiAgent Systems, pages 1620-1628, 2020.

[Zhang et al., 2020c] Wen Zhang, Dengji Zhao, and Yao Zhang. Incentivize diffusion with fair rewards. In 24th European Conference on Artificial Intelligence, ECAI 2020, including 10th Conference on Prestigious Applications of Artificial Intelligence, PAIS 2020, volume 325, pages 251258, 2020.

[Zhang et al., 2020d] Yao Zhang, Xiuzhen Zhang, and Dengji Zhao. Sybil-proof answer querying mechanism. In Proceedings of the 29th International Joint Conference on Artificial Intelligence, pages 422-428, 2020.

[Zhao et al., 2018] Dengji Zhao, Bin Li, Junping Xu, Dong Hao, and Nicholas R. Jennings. Selling multiple items via social networks. In Proceedings of the 17th International Conference on Autonomous Agents and MultiAgent Systems, pages 68-76, 2018.

[Zheng et al., 2020] Yue Zheng, Tianyi Yang, Wen Zhang, and Dengji Zhao. Barter exchange via friends' friends. arXiv preprint arXiv:2010.04933, 2020. 